\title{
La lucha antitracomatosa escolar en Santiago del Estero, Argentina $(1920-1940)^{*}$
}

\author{
The Fight against Trachoma in Schoolchildren \\ in Santiago del Estero, Argentina (1920-1940) \\ A luta antitracromatosa na escola \\ em Santiago del Estero, Argentina (1920-194O)
}

\section{CARLA REYNA ${ }^{* *}$}

Instituto de Estudios para el Desarrollo Social/

Universidad Nacional de Santiago del Estero

(Santiago del Estero, Argentina)

\footnotetext{
* Este artículo forma parte del proyecto de tesis doctoral de la autora, denominado «Tensiones y conflictos en el proceso de surgimiento y de consolidación de la educación sanitaria en Argentina (1900-1983)», dirigido por la Dra. Karina Ramacciotti y el Dr. Alberto Tasso, ambos investigadores del Consejo Nacional de Investigaciones Científicas y Técnicas (CONICET) de Argentina. Artículo de investigación recibido el 16.01.2018 y aceptado el 29.10.2018.

** Becaria doctoral por el Consejo Nacional de Investigaciones Científicas y Técnicas (CONICET), Argentina. Email: reynacarla.e82a@gmail.com
} 


\section{Cómo citar}

REYNA, CARLA (2019). La lucha antitracomatosa escolar en Santiago del Estero, Argentina (1920-1940). Revista CS, 27, 13-35. https://doi.org/10.18046/recs.i26.2703 
Resumen

Abstract

Resumo

Este artículo aborda las prácticas, los sujetos y las instituciones que intervinieron en la lucha antitracomatosa escolar en Santiago del Estero, Argentina, entre las décadas de 1920 y 1940. Los objetivos de este trabajo son, en primer lugar, analizar la conformación de un sistema de atención mixto para preservar la salud visual de los escolares. En segundo lugar, examinar las disputas entre los tracomatólogos locales, de orientación higienista y sanitarista, sobre la etiopatogenia del tracoma, la correlación entre estigmatización étnica y contagiosidad y la efectividad de la campaña escolar en la provincia.

\section{PALABRAS CLAVE:}

saber médico, tracoma, lucha antitracomatosa escolar, estigmatización étnica

This article discusses the practices, individuals and institutions that were involved in the fight against trachoma in school children in Santiago del Estero, Argentina, between the 1920 and 1940s. The goals of this paper are, firstly, to analyze the creation of a mixed health care system to preserve the vision health of schoolchildren. Secondly, to examine the disputes between local trachoma experts, who relied either on a hygienist approach or a sanitary approach regarding the etiopathogenesis of trachoma, the correlation between ethnic stigmatization and contagiousness and the effectiveness of school campaigns in the province.

\section{KEYWORDS:}

Medical Knowledge, Trachoma, Fight against Trachoma in Schoolchildren, Ethnic Stigmatization 
Este artigo trata as práticas, sujeitos e instituições que intervieram na luta antitracromatosa escolar em Santigo del Estero, Argentina, entre as décadas de 1920 e 1940. Os objetivos deste trabalho são, em primeiro lugar, analisar a conformação de um sistema de atenção mista para preservar a saúde visual dos escolares. Em segundo lugar, examinar as disputas entre os tracomatologistas locais de orientação higienista e sanitária sobre a etiopatogenia do tracoma, a correlação entre estigmatização étnica e contagiosidade e a eficácia da campanha escolar na província.

\section{PALAVRAS-CHAVE:}

saber médico, tracoma, luta antitracomatosa escolar, estigmatização étnica 


\section{Introducción}

Este trabajo dista de los clásicos relatos historiográficos que visibilizaron, a comienzos del siglo XX, las transformaciones institucionales derivadas de la inserción de las regiones agropecuarias en el mercado, como Cuyo, el Litoral y Tucumán en el Noroeste Argentino (NOA). Estos espacios sociales se caracterizaron por una activa participación obrero-gremialista, una acelerada diferenciación de sus reparticiones estatales y la formulación temprana de políticas sociales. En contraste, Santiago del Estero, una provincia del NOA, transitó hacia un modelo de producción mixto que conservó algunos rasgos precapitalistas, según el impacto de la explotación forestal y del avance de las vías ferroviarias en el distrito. El paso del ferrocarril favoreció la articulación de un complejo agro-forestal y además impulsó la llegada de inmigrantes -italianos, españoles, sirios y libaneses-, la conformación de comunidades agrícolas y la inversión de capitales (Tasso, 2007). No obstante, esos cambios fueron insuficientes para insertar a la provincia en el modelo agro-exportador. Las magras recaudaciones fiscales, el lento proceso de desarrollo del aparato burocrático y, en consecuencia, una gran incapacidad para atender a las demandas de la población, posibilitaron que las organizaciones benéficas locales monopolizaran la asistencia social hasta la década de 1940, momento en que comenzó a expandirse el poder centralizador del Estado nacional (Biernat, 2015; Tenti, 2013).

En este marco, de cara a las enfermedades invalidantes que pudieran reducir la cantidad de brazos para el trabajo agrícola e incurrieran en mayores cargas sociales, la salud infantil fue una preocupación para las elites dirigentes de la provincia. A tono con el discurso nacionalista de la época, la población escolar debía ser ponderada como futuro capital humano. Desde esta perspectiva, la educación debía consolidar la identidad nacional y preservar la salud para la capacidad productiva y reproductiva de la fuerza laboral (Biernat y Ramacciotti, 2013; Nari, 2004; Shueler y Sotuhwell, 2011; Torres, 2001).

Durante las primeras décadas del siglo XX el afán por inculcar conductas profilácticas en las escuelas argentinas se centró en la salud bucodental, la alimentación, la higiene hogareña, la puericultura, la gimnasia deportiva y la prevención de enfermedades infectocontagiosas. Se transmitieron cortos cinematográficos, diapositivas y conferencias radiotelefónicas, y se distribuyeron cartillas informativas con la pretensión de convertir a los escolares en pequeños agentes de sanidad en el hogar (Álvarez y Reynoso, 2011; Armus, 2007; Biernat y Ramacciotti, 2013; Cammarota, 2011; Lionetti, 2011; Nari, 2004; Ramacciotti, 2009; Ramacciotti, 2010; Ramacciotti y Testa, 2014). 
El repertorio de pautas sanitarias impartidas matizó el perfil epidemiológico de cada región. En el caso de Santiago del Estero, cobró singularidad durante las décadas de 1920 y 1930 la organización de un plan de lucha escolar para erradicar el tracoma -también conocido como conjuntivitis granulosa-, una infección ocular ocasionada por la bacteria chlamydia trachomatis. Aún prevalente a nivel mundial, la afección provoca la formación de granulomas en la conjuntiva y en los párpados que, en estadios avanzados, generan lesiones graves en la córnea como la ceguera parcial o total, en forma irreversible. La infección se transmite por contagio directo, por objetos contaminados y a través de las moscas que estuvieron en contacto con secreciones oculares o nasales de personas infectadas. Aunque la tasa de incidencia es elevada sobre todo en niños pequeños, el modo de prevenirla es a través de la higiene periódica, condicionada por el acceso a agua segura (Organización Mundial de la Salud, 2017).

Si bien los antecedentes mundiales del tracoma como entidad patológica datan desde el siglo XIX, recién en los años 1920, gracias a la incipiente conformación de un cuerpo de saberes médicos interconectados, se pudo identificar el vector del tracoma y, a fines de la década, constatar la presencia de una enfermedad global. De hecho, el flujo bidireccional de las investigaciones entre Argentina y Europa fue una muestra de que los oftalmólogos locales tuvieron un lugar destacado en la asociación internacional de tracomatología respecto de otros países de América Latina, en particular sobre profilaxis y terapéutica. Entre ellos, los representantes argentinos con mayor peso en esta red de expertos fueron los oftalmólogos Enrique Di María y Justo Lijó Pavía, ambos funcionarios del Departamento Nacional de Higiene (DNH) (Di Liscia y Gioia, 2017).

Desde esta última fila de estudio, es reciente la historiografía argentina vinculada a la irrupción de esta afección oftalmológica en el país. En primer lugar, María Di Liscia y María Estela Fernández (2014) investigaron la conexión entre educación e implementación de medidas profilácticas en los ingenios azucareros de la provincia de Tucumán entre 1880 y 1940. Para las autoras, la inmigración ultramarina constituyó el vehículo de ingreso a fines del siglo XIX, cuando el primer caso argentino se registró en 1903. Durante las primeras décadas del siglo XX los registros sobre «tracomatosos» se incrementaron en las provincias del Litoral y del Norte del país, donde se asentó la mayoría de las comunidades extranjeras.

En segundo lugar, Emma Gioia (2016) estudió la recepción de los inmigrantes con tracoma en el Puerto de Buenos Aires durante la primera mitad del siglo XX. Gioia pudo constatar cómo el desarrollo internacional de la tracomatología como especialidad médica, la consolidación de una política transnacional para combatir la patología y el ideario nacionalista local confluyeron en el discurso médico argentino, provocando «una sobrerrepresentación y estigmatización de los inmigrantes», 
particularmente los de origen sirio (Gioia, 2016: 1). En efecto, el porcentaje de extranjeros rechazados por tracoma representó apenas el 0,05\% de los tres millones de inmigrantes que llegaron a la Argentina entre 1908 y 1943 (Gioia, 2016: 7). No obstante, el correlato discursivo entre contagiosidad y etnia incidió en el interés de los médicos locales, que hacia los tempranos años veinte intensificaron sus investigaciones y desarrollaron campañas preventivas en el interior del país, con especial atención en el NOA.

En la provincia norteña de Santiago del Estero, un grupo de médicos locales preocupados por la salud visual infantil fundó en 1928 la Liga contra el Tracoma. Esta asociación, en coordinación con otras organizaciones civiles como la sede provincial de la Cruz Roja Argentina, con agencias públicas locales y nacionales y con la contribución de enfermeras especializadas y de numerosas profesionales del magisterio, implementó un programa escolar -primero en la capital y luego en todo el distrito- orientado a la prevención, la detección y el tratamiento de la conjuntivitis granulosa. Durante la década de 1930, la experiencia santiagueña cobró reconocimiento internacional; sin embargo, la tasa de incidencia, luego de diez años de lucha, no pudo revertirse.

Mediante el examen de las particularidades del plan de erradicación en un contexto extra-céntrico como Santiago del Estero, el presente trabajo pretende contribuir a los trazos iniciales sobre el estudio del tracoma como patología endémica en Argentina (Di Liscia y Fernández, 2014; Di Liscia y Gioia, 2017; Gioia 2016). Asimismo, esta propuesta analítica dialoga con otro corpus de investigaciones recientes, que matizan la complejidad que subyace en la conformación de modelos asistenciales mixtos. Éstos exploran la tensa relación entre asociaciones benéficas y Estado que, en forma complementaria o competitiva, actuaron en el cuidado y atención de la salud en el proceso de expansión del sistema sanitario (González Leandri, 2013; Moreyra, 2009; Ortiz Bergia, 2009, 2015; Suriano y Lvovich, 2006).

Con este propósito, este artículo aborda las prácticas, los sujetos y las instituciones que intervinieron en la lucha antitracomatosa escolar en la provincia, entre las décadas de 1920 y 1940. Analiza, en primer lugar, la conformación de un sistema de atención mixto para preservar la salud visual de los escolares. En segundo lugar, examina las disputas entre los tracomatólogos locales, de orientación higienista y sanitarista, sobre la etiopatogenia del tracoma, la correlación entre estigmatización étnica y contagiosidad y la efectividad de la campaña escolar en la provincia.

El corpus documental es variado. Incluye algunas memorias e informes de miembros de la Liga contra el Tracoma de Santiago del Estero y de la sede local de la Cruz Roja Argentina; y artículos de divulgación científica en la Revista del Comité Internacional de la Cruz Roja y en el Boletín de la Oficina Sanitaria Panamericana. 
En esta investigación se exponen, en primer lugar, el inicio de la política nacional de erradicación del tracoma en Argentina a partir de 1910 y las características que adquiere la lucha antitracomatosa en la provincia de Santiago del Estero. En los subtítulos siguientes se aborda la formulación del plan y la organización de un modelo de intervención mixto para su implementación en la capital santiagueña. Luego, se desarrollan los avances y los retrocesos de la campaña condicionados por la resistencia a los estigmas discriminatorios, la distorsión de las estadísticas y las posiciones encontradas entre los facultativos de la LCT. Por último, se analiza la intervención de la agencia sanitaria nacional para ampliar el plan a todo el territorio provincial y se ausculta la difusión de la experiencia local en las incipientes redes de cooperación sanitaria internacional.

\section{La lucha contra el tracoma: entre la filantropía y la responsabilidad estatal}

Según el Departamento Nacional de Higiene (DNH), el tracoma en el NOA era endémico y registraba la mayor tasa de incidencia nacional. Estos datos provenían del trabajo del prestigioso oftalmólogo argentino Enrique Demaría, quien, en 1910, delegado por la repartición sanitaria, recorrió las provincias de la región para describir las características y la distribución de la patología (Barbieri, 1924). Sin embargo, los registros efectuados se habían limitado a las cercanías de las capitales de cada distrito durante 2 o 3 días, por lo que las estimaciones sobre el perfil de la endemia podían ser ampliamente superadas (Olle, 1933). Esta exploración marcó el inicio de una política para combatir la afección, cuando más tarde la institucionalización de la patología como problema sanitario se formalizó con la creación de la División de Profilaxis del Tracoma y Oftalmías, bajo la órbita del DNH y la dirección del oftalmólogo y miembro de la Cruz Roja Argentina Antonio Barbieri (Di Liscia y Fernández, 2014). A estos efectos, se crearon dispensarios de oftalmías infecciosas en las provincias de Santiago del Estero, Jujuy, Salta y Tucumán (Boletín de la Oficina Sanitaria Panamericana, 1937).

Durante los años veinte y treinta, en el círculo médico argentino dedicado a estudiar la distribución de la patología en el NOA, higienistas y sanitaristas se debatían por atribuir su etiología. Los primeros sostenían que su origen radicaba en la ignorancia y la falta de aseo de los sectores más pobres, que pronto se asoció a la población inmigrante y rural (Álvarez, 1928; 1930). Por su parte, los sanitaristas invertían la mirada y afirmaban que eran las deplorables condiciones de vida y las características climáticas -como el polvo y las altas temperaturas-las que generaban 
un caldo de cultivo para la afección (Cárdenas, 1943; Di Lullo, 1939; Olle, 1933). Estas controversias procedían de las dos vertientes investigativas que, en la década del veinte, se orientaron a la determinación del agente causal y a explorar las medidas preventivas más eficaces. Más allá de exigir condiciones higiénicas, un puñado de médicos comprendía que se necesitaban transformaciones sociales más profundas como el acceso a agua potable y un régimen laboral que superara al patronazgo.

En Santiago del Estero, la incertidumbre sobre las consecuencias irreversibles del tracoma resonó en la elite médica local, preocupada por el margen de población infantil que podría perder la visión y con ello, un importante caudal de futuros trabajadores (Álvarez, 1928). Como se indicó más arriba, las estadísticas de Demaría arrojaron cifras erradas, pero alarmantes, de casos de tracoma. En la capital santiagueña el porcentaje de casos diagnosticados en las escuelas fue del 14\% y en los hospitales alcanzó el 59\%, mientras el estado sanitario del interior provincial representó una incógnita. En el seno de este grupo de facultativos, liderado por el destacado médico Antenor Álvarez, ${ }^{1}$ ex gobernador de la provincia, por entonces titular de la Cruz Roja Argentina de Santiago del Estero (CRA-SDE) y miembro del Consejo de Higiene de la Provincia, surgió la iniciativa de prevenir la ceguera a edades tempranas a través de la educación sanitaria y de la asistencia escolar.

De acuerdo a la tesis formulada por Gioia (2016), el plan de lucha antitracomatosa escolar emprendido en la provincia a partir de 1928 estuvo atravesado por una visión lineal entre salud y nacionalidad, generando un campo propicio para la conformación de estereotipos y de estigmas discriminatorios, que recayeron sobre los inmigrantes del cercano oriente. De hecho, a fines de la década de 1910, los abultados casos relevados en las escuelas fueron asociados a la presencia de menores que provenían de estas familias, razón por la que en las tareas de registro y de clasificación de los escolares «tracomatosos» fue requisito consignar la nacionalidad (Álvarez, 1928).

Sin embargo, según el Censo Nacional de Población de 1914, se registraron en la provincia, bajo la categoría de «otomanos», 1.748 personas de origen sirio-libanés, lo que representó apenas el o,67\% de la población santiagueña. En 1947 esta cifra

1. La actuación pública de Antenor Álvarez en la provincia se trata de un caso paradigmático sobre el poder corporativo de los galenos en los inicios del siglo XX (González Leandri, 1999). Su trayectoria cristalizó el entramado público y privado y la doble pertenencia institucional de muchos profesionales de la elite médica santiagueña que tutelaron en forma paralela agencias oficiales y entidades benéficas. Esta condición les posibilitó su permanencia y jerarquización, como burócratas o legisladores, en el incipiente aparato estatal. Álvarez desempeñó más de cien cargos públicos, entre ellos científicos, administrativos, honoríficos y electivos, que le valieron el prestigio nacional e internacional (Oddo, 1970). Cabe mencionar que fue delegado del Departamento Nacional de Higiene para combatir el paludismo, legislador provincial y nacional, director del Consejo de Higiene de la Provincia, gobernador provincial, presidente de la filial santiagueña de la Sociedad Argentina de Patología Regional del Norte, entre otros. 
ascendió a tan sólo 2.096 individuos, pero la proporción sobre el total de habitantes de la provincia descendió al o,44\% (Tasso, 1988: 71).

\section{La organización de la lucha en la provincia}

De cara a la escasez de médicos, de centros de atención especializada y a la preocupación por la invalidez a edades tempranas, Álvarez fundó la primera escuela de auxiliares de sanidad, destinada exclusivamente a la formación de mujeres. Bajo su presidencia, en 1920 inauguró la filial santiagueña de la Cruz Roja Argentina (CRA-SDE), dos años más tarde comenzó a funcionar la escuela de enfermeras y en 1930 la escuela de samaritanas. La formación en enfermería fue inicialmente de dos años y la de las samaritanas un curso intensivo anual, limitado a «una preparación suficiente de primeros auxilios, medicina preventiva e higiene» (Lozano, 1932: 45).

En sus inicios, ambos cursos impartían nociones sobre enfermedades infecciosas; una especialidad en oftalmías; higiene hogareña y personal; puericultura; nutrición y técnicas culinarias. Entre 1922 y 1944 se inscribieron aproximadamente 500 aspirantes, de las cuales alrededor del 25\% eran docentes (Cruz Roja Argentina de Santiago del Estero, s.f.). Podía advertirse que la construcción de un espacio socio-ocupacional vinculado a lo sanitario había despertado el interés de muchas de las mujeres que, por sus vínculos de parentesco o filiación política, formaban parte del círculo de la elite médica. ${ }^{2}$ Asimismo, las necesidades que se les presentaban a las maestras ligadas a la resolución de cuestiones sanitarias y la demanda de saberes y prácticas habían encontrado un espacio de formación complementaria. La preparación de cuadros técnicos para combatir la conjuntivitis granulosa eran indicios de las limitaciones del DNH para hacer efectiva la política de erradicación a pesar de contar con una repartición específica.

Por su parte, Álvarez formaba parte de una densa red de vínculos a nivel nacional interesada en la nosología de las provincias norteñas. Contaba con la contribución de los oftalmólogos argentinos y miembros de la Cruz Roja Argentina como Antonio Barbieri, Enrique Di María y Rodolfo Olle; figuras representativas en la red internacional de tracomatólogos, interesados en la distribución y en su etiología biológica,

2. En la primera cohorte fue designada como directora de la escuela de enfermeras Carolina Echegaray de Helman, esposa del médico Romualdo Helman, quien la instruyó en nociones básicas de enfermería y cirugía menor. Las primeras inscriptas fueron Delia Santillán Palacio de Álvarez, esposa de Antenor Álvarez; Tomasa Santillán Palacio, cuñada de Álvarez y una de las enfermeras santiagueñas más importantes; Carlota S. de Canal Feijóo, casada con el médico Enrique Canal Feijóo. Véase Páez Luna (1983). 
aún incierta. Álvarez también era titular de la filial santiagueña de la Sociedad de Patología Regional del Norte, presidida por el médico bacteriólogo Salvador Mazza. ${ }^{3}$ Ambos nexos fueron elementales para emprender y coordinar la campaña sanitaria en la región.

En 1928, con motivo de la inauguración del Curatorio de Oftalmías dependiente de la CRA-SDE en la Escuela del Centenario, Álvarez presentó en su discurso inaugural el «Plan de Defensa contra el Tracoma en el Norte Argentino». Este ambicioso programa preveía la conformación de comisiones de higiene y terapéutica, la creación de pequeños dispensarios anti-tracomatosos y la instalación de baños públicos en las poblaciones más afectadas por la falta de agua. La intervención desde las escuelas se haría primero mediante el examen médico al iniciar el año lectivo y luego mediante el aislamiento de los niños infectados en escuelas especiales para «granulosos», las cuales, ante la crónica escasez de recursos estatales, se tornaban inviables. ${ }^{4}$ En este caso, debían asumirse otras medidas como el lavado de manos en el ingreso al aula, la ubicación de los indemnes en pupitres alejados de los infectados e inmigrantes, el uso exclusivo de útiles y libros escolares y la recomendación a las familias de la examinación de la «servidumbre (...); pues, generalmente es tracomatosa» (Álvarez, 1928: 10). El plan formulado por Álvarez denotaba así que los estereotipos portadores de la enfermedad entrelazaban pobreza, ruralidad y etnia.

A los efectos de su implementación, se fundó en ese mismo año la Liga contra el Tracoma (LCT), en principio una asociación civil dependiente de la CRA-SDE, conformada por hombres y mujeres de la corporación médica santiagueña. Pronto, el organismo amplió sus horizontes y se estableció como una entidad regional, integrada también por delegados de las provincias argentinas de Salta, Jujuy, Tucumán y Catamarca (Álvarez, 1928). Si bien aspiraban a intervenir en un amplio marco territorial donde la presencia del DNH era casi nula, en Santiago del Estero inicialmente la campaña tuvo lugar en las escuelas capitalinas.

Las maestras, las enfermeras y las samaritanas especializadas en oftalmías de la CRA-SDE fueron el soporte estructural del plan. Conforme a su deber patriótico y humanitario, las docentes fueron designadas para la detección de casos sospe-

3. La filial santiagueña de la Sociedad Argentina de Patología Regional del Norte fue fundada por Salvador Mazza en noviembre de 1926. La cuarta reunión de esta sociedad fue celebrada entre el 7 y 9 de mayo de 1928 en la capital santiagueña con cada uno de sus referentes provinciales -Jujuy, Salta, Tucumán y Santiago del Estero-. Del mismo modo, la octava reunión de esta sociedad fue realizada entre el 2 y 3 de octubre de 1932 en Santiago del Estero (Oddo, 1999).

4. La primera y única escuela estatal para niños «tracomatosos» fue fundada en 1920 en la provincia de Tucumán, Argentina. Para un estudio más profundo véase Di Liscia y Fernández (2014). 
chosos y para impartir educación preventiva en las escuelas. Por un lado, debían identificar las alteraciones patológicas de los alumnos mediante la observación de signos clínicos como la capacidad visual, la secreción y el enrojecimiento ocular, la inflamación palpebral o la fobia a la luz. Por otra parte, la prescripción educativa debía instalar la peligrosidad del tracoma entre la población, mediante opúsculos, conferencias y proyecciones cinematográficas. La mejor profilaxis para los infantes era «educarlos enseñándoles a aislar sus ojos, como se enseña a los tuberculosos a aislar su boca, convenciéndolos de que no deben toser y escupir» (Álvarez, 1928: 6).

El hogar era territorio de las enfermeras y las samaritanas, encargadas de la inspección y el tratamiento en la escuela y en las viviendas. Por su especialidad en oftalmías, estas mujeres dispensaban los pequeños consultorios escolares para atender a los niños derivados por las docentes, a sus familias y a los pobladores de la zona que lo requerían. Los casos graves eran atendidos por el oculista Rodolfo Olle, a cargo de los servicios oftalmológicos de la CRA-SDE, del Hospital Mixto y del Hospital Independencia de la provincia, que comenzaron a funcionar a partir de 1929 (Álvarez, 1941; Lozano, 1932; Olle, 1934).

Así, la estrategia profiláctica consistía en la atomización de la patología y en la reificación y segregación de los «granulosos». La secuencia del diagnóstico y tratamiento de los cuadros leves implicaba una distribución genérica del trabajo sanitario, donde la falta de recursos estatales y de personal se tradujo en actividades de cuidado no remuneradas, derivadas del deber patriótico de las maestras y del espíritu «samaritano» de las enfermeras. Por un lado, tomando los aportes de Estela Grassi (1989), en la campaña antitracomatosa los costos de la atención se transfirieron al trabajo ad honorem de estas mujeres, en función de su rol social asignado. Por otro lado, en referencia a los mecanismos de legitimación de la experticia en el aparato burocrático y en la comunidad internacional (Plotkin y Zimmerman, 2012), la doble pertenencia institucional -estatal y civil-y la segregación de tareas sanitarias por género resultaron, al menos en esta experiencia sanitaria, necesarias para la realización de investigaciones en sus dominios de conocimiento. Como se analiza en el próximo apartado, el registro estadístico, el diagnóstico y el tratamiento que efectuaron estas mujeres a los escolares, en el seno de una organización filantrópica, constituyeron los insumos para que éstos célebres médicos publicaran artículos, dictaran conferencias y divulgaran sus trabajos en el extranjero. 


\section{Retrocesos y avances de la campaña antitracomatosa}

Durante los primeros tres años de la campaña, la resistencia opuesta a los estigmas discriminatorios, la multiplicidad de registros, la distorsión de las estadísticas y las estrategias retóricas para validar o impugnar el éxito del plan, obstaculizaron su alcance.

En primer lugar, a pesar de la mediación de algunas asociaciones vecinales, en las recorridas barriales las familias se oponían a la inspección de sus viviendas. Según el primer informe trienal 1928-1931 de Olle (1931), a cargo de la sección de las escuelas superiores del plan, también las autoridades escolares y los padres se mostraban reticentes a que los niños fueran examinados. Las actitudes manifiestas dejaban entrever las resistencias a ser estigmatizados como «tracomatosos», pues implicaba la segregación escolar y el aislamiento del niño y su familia en sus viviendas.

Estos obstáculos generaron una reorganización de las tareas del plan de erradicación. Las maestras comenzaron a registrar a los escolares «tracomatosos» $\mathrm{y}$ «sospechosos» mediante siglas clasificatorias, según nacionalidad y número de alteraciones visuales observadas. En línea con lo postulado por Gioia (2016), en Santiago del Estero la identificación de niños extranjeros y descendientes de inmigrantes se trató de un criterio de codificación derivado de la asociación entre contagiosidad y etnia. Las nacionalidades tuvieron consignación obligatoria y, para evitar resistencias, los alumnos no eran notificados sobre su diagnóstico.

Por su parte, las autoridades escolares estaban a cargo de la sensibilización de los padres sobre la importancia de la examinación y de las curaciones en los niños, nociones que fueron reforzadas con la vulgarización de las medidas de prevención y tratamiento de la enfermedad. Los primeros avances del cambio de estrategia se reflejaron en la conducta de las familias en la vigilancia del tratamiento y en las demandas para atender a sus hijos no escolarizados.

Para Olle, la experiencia les había «(...) enseñado que no es necesario aislar a estos enfermos [dado que] su aislamiento se consigue inculcando y divulgando hábitos de higiene social e individual» (Olle, 1931:26). Estos indicios señalaban que el avance de la campaña dependía no sólo de la intervención de médicos, maestras y enfermeras en esos ojos, sino también de la medida en que la población se convirtió en usuaria de estos servicios al imponer sus propios marcos simbólicos (Bourdieu, 1997), negociando la des-estigmatización social por la asistencia gratuita y la implementación de consejos sanitarios.

No obstante, respecto a estos logros emergieron posiciones encontradas entre los propios facultativos de la LCT. La multiplicidad de registros y de estadísticas acrecentó esta disparidad y morigeró los alcances de la lucha antitracomatosa. En esta línea, los estudios de Claudia Daniel (2012) dan cuenta de las diversas limitaciones 
que el DNH tuvo hasta los años cuarenta para cuantificar y predecir la evolución epidemiológica de las patologías. Más precisamente, estas dificultades se vincularon a la aplicación de los instrumentos estadísticos y a los métodos de interpretación de los datos, construidos en base a estándares de categorización y nomenclatura internacionales. Asimismo, la proliferación y el solapamiento de trabajos estadísticos financiados con recursos privados y estatales tornaron disímiles y fragmentadas las cifras oficiales.

Dentro de esta dinámica, la disparidad de los cómputos fue visible en los informes elevados por distintos miembros de la LCT, confrontados con las cifras divulgadas sobre esta campaña en revistas de circulación académica nacional y con las citas y referencias del Boletín Oficial de la Oficina Panamericana de la Salud y la Revista del Comité Internacional de la Cruz Roja durante la década del treinta.

Álvarez, como referente argentino del comité, destacaba que en 1930 se examinaron en 26 escuelas capitalinas a un total de 6.752 niños, el 14,72\% fue diagnosticado con tracoma. Sin embargo, este porcentaje oscilaba: en los establecimientos periféricos alcanzaba un $35 \%$, mientras que en los más céntricos descendía a un $4 \%$. Las enfermeras administraban medicinas a los escolares cada 48 horas y realizaban en promedio 450 curaciones diarias (Revista y Boletín de la Sociedad de la Cruz Roja, 1931; 1932; Boletín de la Oficina Sanitaria Panamericana, 1932; 1935; 1937). En estas reseñas, la campaña antitracomatosa se consignaba como plan modélico, sin precedentes y exitoso.

En cambio, para Olle (1931), los cómputos iniciales arrojaban que la campaña solo había tenido efectos paliativos: la morbilidad había disminuido de un $14 \%$ inicial a un $10,8 \%$. Si tomamos como referencia que la campaña se implementó solo en la capital, el reducido nivel de alfabetización en la provincia y los patrones migratorios de las familias rurales, ${ }^{5}$ estas cifras representaron una estimación y pudieron ser superadas ampliamente. Asimismo, este dato demostraba la baja incidencia de los niños descendientes de inmigrantes sobre el total de casos de tracoma infantil. El porcentaje de estos escolares oscilaba entre el 17\% y el 23\%, encabezado por italianos, españoles, sirios y libaneses (Olle, 1931:11-19). Por un lado, estos resultados sugerían que los principales hogares afectados se ubicaban en los suburbios, en condiciones críticas de existencia. Por otro lado, de acuerdo a la tesis de Emma Gioia (2016), los

5. Los ingenios azucareros tucumanos constituían fuentes laborales provisorias para los campesinos santiagueños cuando la sequía limitaba la siembra de sus campos. Durante la cosecha, los «zafreros» migraban desde Santiago del Estero hacia Tucumán, muchos de ellos con sus familias para incrementar la mano de obra - sin discriminación de sexo ni edad- y conseguir mayores ingresos. Esta condición de «trabajadores golondrinas» los exponía a situaciones extremas de hacinamiento al convivir con otras familias, en malas condiciones higiénicas y con carencias nutricionales. 
casos registrados demostraban que estos inmigrantes fueron sobrerrepresentados como responsables de la difusión de la patología en la provincia.

Así lo demostraban las estadísticas presentadas por Olle en el V Congreso Nacional de Medicina (1934). El relevamiento que realizó durante su labor en los servicios de oftalmología públicos y privados, indicó que la mayoría de los enfermos de tracoma eran nativos y las formas observadas benignas, sin complicaciones graves (Olle, 1934). ${ }^{6}$

Las incongruencias entre cifras oficiales y particulares hacían foco en la capital, mientras en el interior provincial la incidencia del tracoma era una incógnita y, por tanto, era considerado el reservorio de la patología. Se estimaba que la afección podía ascender en las escuelas rurales hasta el 60\% (Olle, 1934: 655). Ante la ausencia en los distritos rurales de oftalmólogos y de enfermeras, las maestras fueron exhortadas esta vez no tan solo para la educación preventiva, sino también para las curaciones.

Con este propósito, en 1933 Álvarez, nuevamente titular del Consejo de Higiene Provincial, elevó al poder ejecutivo local el plan «Asistencia del Tracoma en Santiago del Estero». De acuerdo a la experiencia de países europeos, se capacitarían docentes en terapéutica oftalmológica. Las nuevas disposiciones establecían en forma obligatoria que las maestras debían administrar el tratamiento a los niños de la división a su cargo antes de finalizar la jornada escolar. La propuesta no incurriría en gastos presupuestarios, dado que el trabajo de las docentes sería «el medio más económico y eficaz que impone el patriotismo» (Álvarez, 1933: 4). El plan de asistencia también preveía la ampliación del consultorio de oftalmología del Hospital Independencia a una sala de internación, debido a que hasta entonces la construcción de un centro nacional especializado no se había concretado en la provincia.

Un decreto del gobierno provincial aprobó inmediatamente la propuesta, contemplando la acción conjunta de las autoridades escolares, sanitarias y las instituciones privadas de asistencia social. Ese mismo año, el plan fue adoptado como política oficial en la Primera Conferencia Nacional de Asistencia Social (Álvarez, 1934) y alcanzó repercusión internacional a través del Boletín de la Oficina Sanitaria Panamericana (1935).

6. Las estadísticas suministradas por Olle correspondían a 10.0oo pacientes atendidos en los servicios de oftalmología del Hospital Independencia, de la LCT y de su consultorio particular, y a 18.ooo niños examinados en las escuelas capitalinas a través de la intervención de la LCT. El registro se había efectuado entre 1928 y 1933. Olle estimaba que el 60\% de los atendidos en los consultorios eran por conjuntivitis de diferente etiología, propiciadas por el calor, el polvo y el viento. En efecto, los casos recrudecían en agosto cuando comenzaba a correr el viento zonda hasta finalizar el período estival. 


\section{La intervención estatal en la lucha privada}

Si bien una de las estrategias del proceso de centralización del DNH fue el desplazamiento de las organizaciones benéficas (Biernat, 2015), las dificultades técnicas y presupuestarias en Santiago del Estero generaron que ambas esferas, pública y privada, continuaran complementándose para atender las demandas sanitarias de la población, pero en forma subordinada. No obstante, muchas de estas iniciativas se tornaron estériles cuando la repartición suspendió la provisión de recursos al despuntar la década del cuarenta.

Estas intermitencias burocráticas fueron evidentes a partir de 1937, cuando la campaña antitracomatosa fue coordinada por la CRA-SDE, la LCT y la Sección Oftalmológica de Profilaxis del Tracoma del DNH, que por entonces era presidido por Miguel Sussini (1932-1938). El nuevo plan dispuso la funcionalidad de cuatro dispensarios oftalmológicos dependientes de esta sección y la subdivisión del distrito en 6 secciones, cada una a cargo de un inspector médico. En ese mismo año, bajo la dirección de Alberto Cárdenas, oftalmólogo de la CRA-SDE y de la LCT, se examinaron a 49.626 niños en 560 escuelas del interior (Imagen 1 y 2).

Las estadísticas indicaban que la tasa de incidencia era estable comparada con la de diez años atrás: el 14\% había sido diagnosticado con la patología. Aunque la intervención del DNH logró ampliar el horizonte del plan de erradicación, estas acciones se diluyeron cuando la repartición se retiró de la campaña provincial en 1941 y sólo continuó la LCT (Cárdenas, 1943). Estos eran indicios, por un lado, del influjo de los vaivenes políticos y del recambio de los elencos burocráticos en la caída presupuestaria (Biernat, 2015; Ramacciotti, 2009). Por otro lado, de que las medidas implementadas no tendrían éxito si no se mejoraban las condiciones de vida de la población.

Las incongruencias estadísticas y etiológicas continuaron en el centro de los debates médicos acerca del cuadro endémico en Santiago del Estero. Como presidente de la delegación santiagueña de la Sociedad de Patología Regional del Norte, en 1941 Antenor Álvarez fue designado para confeccionar el mapa nosológico y el catastro sanitario de la provincia. En esta obra, el higienista consideraba las enfermedades endémicas, como el tracoma, independientes del clima y de los determinantes sociales (Álvarez, 1941).

Tanto para Orestes Di Lullo (1939) como para Álvarez (1941), ambos integrantes de la LCT, a fines de la década de 1930 la lucha antitracomatosa había erradicado la patología en la provincia. Estos pronunciamientos contrastaban con lo relevado por Cárdenas que, ante el retiro del DNH, continuó en la campaña de la asociación. En 
1943, sólo en las escuelas del distrito capitalino, se confirmaron 1.026 casos de un total de 7.891 alumnos (13\%) (Cárdenas, 1943).

Para entonces, el campo de la tracomatología ya había trazado algunos avances. Las investigaciones e intercambios entre oftalmólogos argentinos y especialistas europeos derivaron en el uso de terapias antimicrobianas a principios de los años 1940, tales como el empleo de sulfamidas y de antibióticos, con amplios beneficios para quienes padecían la patología (Di Liscia y Gioia, 2017). No obstante, la permanencia de las desfavorables condiciones de vida en Santiago del Estero y la crónica falta de recursos estatales tornaron impredecible el control de la endemia.

\section{Conclusiones}

Como señalan Di Liscia y Gioia (2017:30), el tracoma representó «un problema no solo sanitario, sino también productivo y de seguridad».

El plan formulado por Antenor Álvarez cristalizó esta construcción simbólica. Los estereotipos portadores de la enfermedad entrelazaron pobreza, ruralidad y etnia. Inicialmente, los inmigrantes sirios y libaneses fueron representados como los responsables de la difusión de la conjuntivitis granulosa. Luego de más de una década de lucha antitracomatosa en la provincia, la elevada proporción de casos autóctonos, con particular atención a los espacios rurales privados de agua potable, demostró la baja incidencia de estas colectividades en el desarrollo de un problema endémico. Como se señaló, hasta 1947 la comunidad sirio-libanesa no superó el 1\% en relación al total de la población santiagueña.

Asimismo, cabe destacar las conclusiones de Rodolfo Olle respecto de las características clínicas de la conjuntivitis granulosa observadas en los individuos nativos. Según el oftalmólogo, de los 10.000 casos relevados, la mayoría fueron cuadros benignos que no comprometían la capacidad visual. Entre la comunidad de oftalmólogos argentinos y los propios miembros de la LCT, estos hallazgos se contraponían al discurso generalizado sobre la peligrosidad del tracoma y la propagación de la enfermedad a causa de la inmigración.

Sin duda, la excepcionalidad de la campaña en un contexto extra-céntrico como Santiago del Estero se concretó mediante la distribución genérica del trabajo sanitario y la vinculación de los galenos locales con redes médicas de investigación regional y multinacional y con asociaciones filantrópicas de alcance global, como el Comité Internacional de la Cruz Roja. 
En primer lugar, fue evidente que la falta de recursos estatales y de personal se tradujo en actividades de cuidado no remuneradas, derivadas del deber patriótico de las maestras y del espíritu «samaritano» de las enfermeras, de modo que los costos de la atención se transfirieron al trabajo ad honorem de estas mujeres profesionales.

En segundo lugar, dentro de este entramado institucional se verifica que el registro estadístico, el diagnóstico y el tratamiento que efectuaron estas profesionales a los escolares, en el seno de una organización filantrópica, constituyeron los insumos para que los médicos que encabezaron la campaña publicaran artículos, dictaran conferencias y divulgaran sus trabajos en el extranjero. Así, la doble pertenencia institucional, como filántropos y como titulares de servicios públicos, constituyó un mecanismo de legitimación de experticia en el plano nacional e internacional. Un accionar de este tipo describe la trayectoria de Álvarez. Sus nexos con la Sociedad de Patología Regional del Norte, con la Cruz Roja Argentina y con el círculo de oftalmólogos locales-como Enrique Demaría y Antonio Barbieri, quienes, además de ser funcionarios públicos, también tutelaron dependencias de la Cruz Roja-fueron las puertas de acceso para internacionalizar, a través del Boletín de la Oficina Sanitaria Panamericana y de la Revista del Comité Internacional de la Cruz Roja, la experiencia realizada en Santiago del Estero. Los resultados de la campaña comenzaron a publicarse en la década de 1930, a pesar de las incongruencias estadísticas que ponían en duda la eficacia del plan.

Si bien la extensión del presente artículo limita el abordaje del influjo de la campaña antitracomatosa santiagueña en la configuración de modelos institucionales similares en otras regiones o países, en este análisis se pudo comprobar su circulación como antecedente latinoamericano para combatir la patología, en el marco de incipientes redes de cooperación sanitaria internacional.

Se propone entonces una nueva agenda investigativa. Será objeto de futuras investigaciones explorar el influjo de otras escalas sobre la conformación de políticas sanitarias transnacionales. Un estudio que avanza en los trazos iniciales de este campo es el de Julien Comte (2008). Su trabajo sobre la configuración de las primeras políticas para controlar la sífilis en París, Buenos Aires y Nueva York entre 1900 y 1940, evidencia el entrecruzamiento de saberes médicos entre América y Europa. En este sentido, advierte sobre la posibilidad de un esquema transatlántico no sólo en dirección norte-norte y norte-sur: es necesario visibilizar el peso de las ideas de América Latina en la dirección inversa, tomando como referencia que los procesos locales son expresiones de fenómenos globales y viceversa. 


\section{Referencias}

ÁLVAREZ, A. (1928). Plan de defensa contra el tracoma en el Norte Argentino. Santiago del Estero: Tipografía R. Risas.

(1933). Asistencia del Tracoma en Santiago del Estero. Plan de Defensa sancionado por el Consejo de Higiene Pública y aprobado por el Poder Ejecutivo de la Provincia. Santiago del Estero: s.i.

(1934). Asistencia Social del Tracoma en la República Argentina. Ponencia presentada en la Primera Conferencia Nacional de Asistencia Social. Buenos Aires: Casa Jacobo Peuser.

(1941). Santiago del Estero, ciudad de invierno. Santiago del Estero: Talleres Gráficos El Liberal.

ÁLVAREZ, A. y REYNOSO, D. (2011). Entre el abandono y la debilidad. El cuidado de la salud en la primera infancia, entre 1920-1930. En I. Cosse, V. Llobet, C. Villalta y M. Zapiola (comps.), Infancias: politicasy saberes en la Argentinay Brasil (siglos XIX y XX) (pp. 123-149). Buenos Aires: Teseo.

ARMUSS, D. (2007). La ciudad impura. Salud, tuberculosis y cultura en Buenos Aires, 1870-1950. Buenos Aires: Edhasa.

BARBIERI, A. (1924). El tracoma en la República Argentina. Anales del Departamento Nacional de Higiene, (30), 20-56.

BIERNAT, C. (2015). El proceso de centralización del Departamento Nacional de Higiene (18801944). En C. Biernat; K. Ramacciotti y J. M. Cerdá (dirs.), La salud pública y la enfermería en la Argentina (pp. 47-83) Bernal: Universidad Nacional de Quilmes Editorial.

BIERNAT, C. y RAMACCIOTTI, K. (comps.) (2013). Crecer y multiplicarse. La política sanitaria materno-infantil, Argentina, 190o-196o. Buenos Aires: Biblos.

BOLETÍN DE LA OFICINA SANITARIA PANAMERICANA (1932). Tracoma, 11 (12), 1289-1294. Recuperado de http://iris.paho.org/xmlui/handle/123456789/10330

BOLETÍN DE LA OFICINA SANITARIA PANAMERICANA (1935). Tracoma y ceguera, 14 (8), 763-769. Recuperado de http://iris.paho.org/xmlui/handle/123456789/18254

BOLETÍN DE LA OFICINA SANITARIA PANAMERICANA (1937). Tracoma y ceguera, 16 (4), 359-363. Recuperado de http://iris.paho.org/xmlui/handle/123456789/16309

BOURDIEU, P. (1997). Razones prácticas. Sobre la teoría de la acción. Barcelona: Anagrama.

CAMMAROTA, A. (2011). El cuidado de la salud de los escolares en la provincia de Buenos Aires durante el primer peronismo (1946-1955). Las libretas sanitarias, las fichas de salud y las cédulas escolares. Propuesta educativa, (35), 113-120.

CÁRDENAS, A. (1943). La lucha antitracomatosa en Santiago del Estero. Buenos Aires: Liga contra el Tracoma en el Norte Argentino. 
COMTE, J. (2008). Syphilis and sex: transatlantic medicine and public health in Argentina and the United States (1900-1940) (Tesis doctoral). University of Pittsburgh, Pittsburgh, Estados Unidos.

CRUZ ROJA ARGENTINA DE SANTIAGO DEL ESTERO (s.f.). Acta de exámenes de enfermeros: 1922-1951, libro de actas $N^{\circ}$ 1. Santiago del Estero: s.i.

DANIEL, C. (2012). Contar para curar. Estadísticas y comunidad médica en Argentina, 1880-1940. História, Ciências, Saúde - Manguinhos, 1 (19), 89-114.

DI LISCIA, M. y FERNÁNDEZ, M. (2014). ¿Separados o juntos? Contagio, escolaridad y tracoma en Argentina (1884-1940). Educar em Revista, (54), 97-121.

DI LISCIA, M. y GIOIA, E. (2017). La investigación sobre el tracoma y las redes internacionales en Argentina (finales del siglo XIX hasta la década de 1930). Anuario de la Facultad de Ciencias Humanas, Universidad Nacional de La Pampa, 13 (13), 17-32.

DI LULLO, O. (1939). La SAN. ASIS. (Ensayo de organización de la sanidady asistencia social). Buenos Aires: Compañía Impresora Argentina.

GIOIA, E. (2016). La salud al poder. Tracoma y políticas migratorias argentinas en la primera mitad del siglo XX. Quinto Sol, 20 (2), 1-24.

GONZÁLEZ LEANDRI, R. (1999). Curar, persuadir, gobernar. La construcción histórica de la profesión médica en Buenos Aires 1852-1886. Madrid: Biblioteca de Historia de América/CSIC.

(2013). Internacionalidad, higiene y cuestión social en Buenos Aires (1850-1910). Tres momentos históricos. Revista de Indias, 73 (257), 23-54.

GRASSI, E. (1989). La mujer y la profesión de asistente social: el control de la vida cotidiana. Buenos Aires: Humanitas.

LIONETTI, L. (2011). Infancia y educación en diálogo: un campo de posibilidades para la reflexión teórica y renovadas perspectivas de análisis. En I. Cosse, V. Llobet, C. Villalta y M. Zapiola (comps.), Infancias: políticas y saberes en la Argentina y Brasil (siglos XIX y XX) (pp. 36-49). Buenos Aires: Teseo.

LOZANO, N. (dir.) (1932). El Libro de la Cruz Roja Argentina. Buenos Aires: Talleres Gráficos de la Compañía General Fabril Financiera.

MOREYRA, B. (2009). Cuestión socialy políticas sociales en la Argentina. La modernidad periférica. Córdoba, 19oo-193o. Bernal: Universidad Nacional de Quilmes Editorial.

NARI, M. (2004). Políticas de maternidad y maternalismo político. Buenos Aires: Biblos.

ODDO, V. (1970). Dr. Antenor Álvarez. Vida yobra de un médico santiagueño precursor del sanitarismo moderno. Santiago del Estero: Taller Imprenta El Liberal. 
(1999). Historia de la medicina en Santiago del Estero. Santiago del Estero: Editorial El Liberal.

OLLE, R. (1931). Tres años de lucha antitracomatosa en las escuelas de Santiago del Estero. Santiago del Estero: Publicación oficial de la Liga contra el Tracoma.

(1934). Las oftalmías infecciosas en Santiago del Estero. En V Congreso Nacional de Medicina. Actas y trabajos (pp. 643-655). Rosario, Argentina: s.i. Ésta es la ciudad en donde se realizó el Congreso. No tiene editorial.

ORGANIZACIÓN MUNDIAL DE LA SALUD (2017). Tracoma. Nota descriptiva. Recuperado de http://www.who.int/mediacentre/factsheets/fs382/es/

ORTIZ BERGIA, M. (2009). De caridades y derechos. La construcción de políticas sociales en el Interior argentino. Córdoba 1930-1945. Córdoba: Centro de Estudios Históricos Profesor Carlos S. A. Segreti.

(2015). El Estado en el interior nacional en la primera mitad del siglo XX. Aproximaciones historiográficas a un objeto en constante revisión. Estudios sociales del Estado, 1 (1), 59-85.

PÁEZ LUNA, J. (1983). Mujeres de la Cruz Roja. Inédito.

PLOTKIN, M. y ZIMMERMAN, E. (comps.)(2012). Los saberes del Estado. Buenos Aires: Edhasa.

RAMACCIOTTI, K. (2009). La política sanitaria del peronismo. Buenos Aires: Biblos.

(2010). De chico, el árbol, se puede enderezar. La salud infantil durante el peronismo. En L. Lionetti y D. Míguez (comps.), Las infancias en la historia argentina (1890-1960). Intersecciones entre prácticas, discursos e instituciones (pp. 175-198). Rosario: Prohistoria.

RAMACCIOTTI, K. y TESTA, D. (2014). La niñez «anormal». Discurso médico sobre la infancia, 1900-1950. Revista Inclusiones, vol. especial (1), 226-248.

REVISTA Y BOLETÍN INFORMATIVO DE LA SOCIEDAD DE LA CRUZ ROJA (1931). (347).

REVISTA Y BOLETÍN INFORMATIVO DE LA SOCIEDAD DE LA CRUZ ROJA (1932). (232).

SHUELER, A. DE y SOUTHWELL, M. (2011). Formación del Estado nacional y constitución de los cuerpos docentes: profesionalización del magisterio en Brasil y Argentina en perspectiva comparada (1820-200o). En D. Vidal, D. y A. Ascolani (coords.), Reformas educativas en Brasily en Argentina. Ensayos de historia comparada de la educación (1820-200o) (pp.147-188.) Buenos Aires: Biblos.

SURIANO, J. y LVOVICH, D. (2006). Las políticas sociales en perspectiva histórica. Argentina $1870-$ 1952. Buenos Aires: Prometeo.

TASSO, A. (1988). Aventura, trabajo y poder. Sirios y libaneses en Santiago del Estero (1880-1980). Buenos Aires: Ediciones Índice. 
(2007). Ferrocarril, quebracho y alfalfa. Un ciclo de agricultura capitalista en Santiago del Estero, 1870-1940. Córdoba: Alción.

TENTI, M. (2013). La formación de un Estado periférico. Santiago del Estero (1875-1916). Santiago del Estero: Ediciones Universidad Católica de Santiago del Estero.

TORRES, C. (2001). Grandezas y miserias de la educación latinoamericana del siglo XX. En Torres, C. (Comp.), Paulo Freire y la agenda de la educación latinoamericana en el siglo XXI (pp. 23-52). Buenos Aires: CLACSO.

\section{Documentos Oficiales}

ARCHIVO GENERAL DE LA NACIÓN. Dpto. Doc. Fotográficos. Buenos Aires. Argentina. «Médico y vacunador en el colegio de Añatuya vacunando y revisando la vista a los alumnos de la misma (Santiago del Estero)», Inventario 206264.

ARCHIVO GENERAL DE LA NACIÓN. Dpto. Doc. Fotográficos. Buenos Aires. Argentina. «Campaña antitracomatosa», Inventario 197583.

\begin{tabular}{l|l} 
ANEXO 1 & Médico y vacunador en el colegio de Añatuya vacunando y revisando la
\end{tabular} vista a los alumnos de la misma (Santiago del Estero)

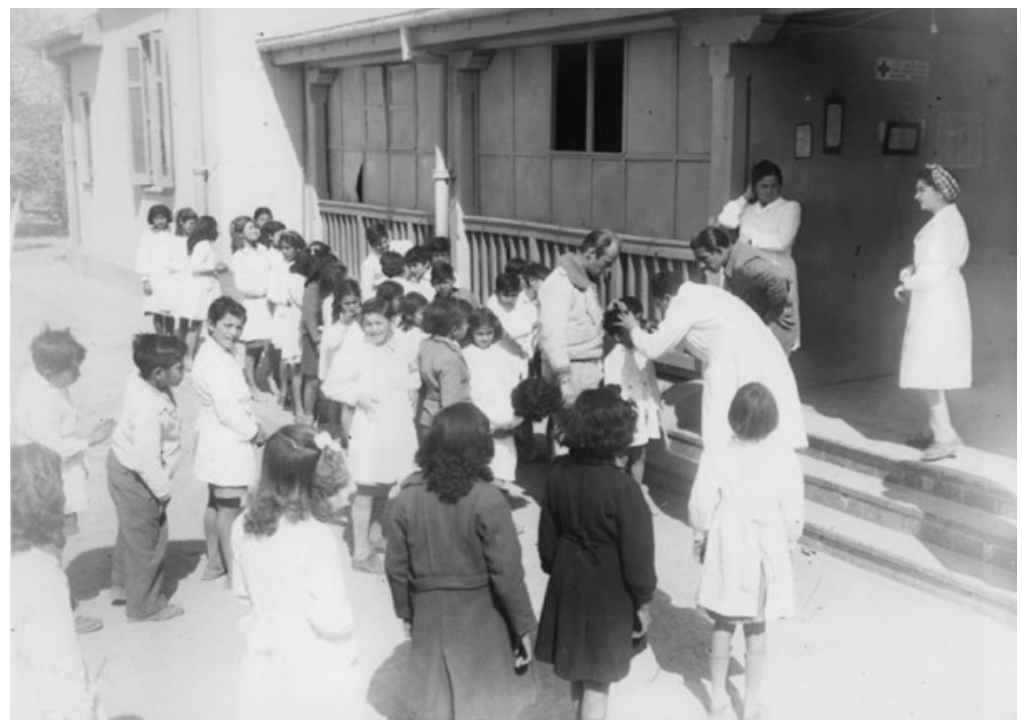

Fuente: Archivo General de la Nación. Dpto. Doc. Fotográficos. Buenos Aires. Argentina. 


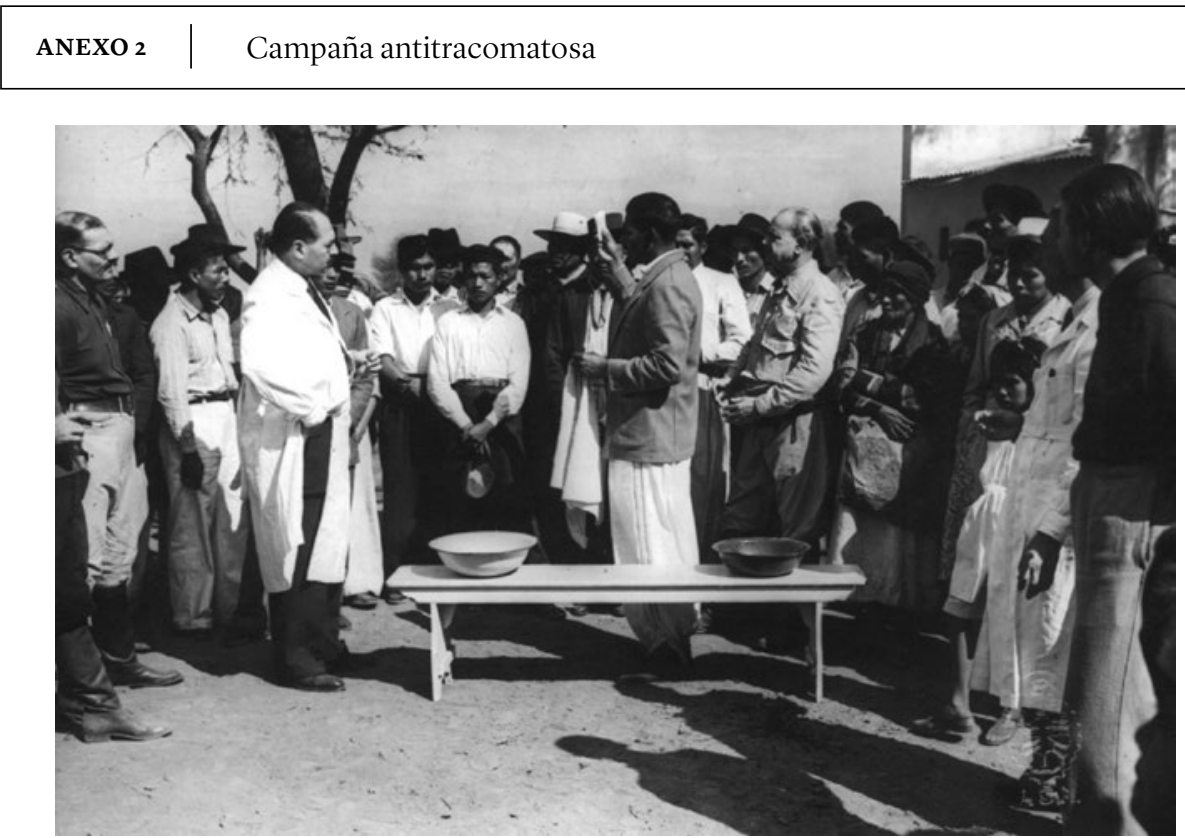

Archivo General de la Nación. Dpto. Doc. Fotográficos. Buenos Aires. Argentina. 